\title{
THERAPEUTIC EFFICACY OF ATTENUATED HIGH VOLTAGE HEALTHTRON DEVICE ON CHILDREN WITH CEREBRAL PALSY
}

\author{
Journal website at; \\ http://mrtbjournal.org/index.php/njmr/issue/current/showToc \\ ${ }^{1}$ OBAOWOEYE, ${ }^{2}$ EOTEMIYE \\ Department of ${ }^{1}$ Physiotherapy and ${ }^{2}$ Paediatrics, Faculty of Clinical Sciences, \\ College of Medicine, University of Lagos, Lagos, Nigeria. \\ Correspondence to: Oba \\ Owoeye \\ toyosialexowoeye@yahoo.com \\ Tel: +234-703-260-2437
}

\begin{abstract}
SUMMARY
Background: Exercise therapy in the form of passive mobilisation and functional training is the most common physiotherapy modality used in improving motor function and modulating spasticity in children with CP. However, modulating spasticity in CP patients remains a huge clinical challenge to physiotherapists and caregivers. The Healthtron device (HD) is an attenuated high voltage electrotherapy gadget claimed to have the ability of modulating spasticity and improving function in children with $\mathrm{CP}$.
\end{abstract}

Objective: To investigate the therapeutic efficacy of the $\mathrm{HD}$ in improving motor function and modulating spasticity in children with $\mathrm{CP}$.

Methods: A randomised controlled study in which 24 children with CP of age 1.310 .0 years having gross motor function classification score (GMFCS) III to V were put in either a Healthtron device (HD) or control (CON) group. The HD group had conventional physiotherapy and the HD treatment while the CON group had conventional physiotherapy alone. Participants in both groups had a total of 12 treatment sessions of 3 times a week for 4 weeks. Outcomes were assessed pre and post-intervention using the gross motor function measure-66 (GMFM-66) and modified asworth scale (MAS) to determine any therapeutic effects of the HD on participants.

Results: Significant improvement was noted in the GMFM66 and the MAS in the HD and CON groups $(\mathrm{p}<0.05)$, except in the MAS measurement for the CON group $(\mathrm{p}=0.083)$. A significantly superior improvement was found in the HD group over the CON group for both outcome measures especially in the modulation of spasticity $(\mathrm{p}<0.001)$.

Conclusion: Attenuated high voltage current generated from the HD combined with conventional physiotherapy resulted in a significant improvement in gross motor function and reduction in spasticity in children with $\mathrm{CP}$. The HD may serve as an adjunct electrotherapy device with other physiotherapy modalities in improving function and modulating spasticity in children with $\mathrm{CP}$. We recommend that laboratory studies be carried out to fully understand the physiological and biochemical effects of the HD on the human body.

Key Words: Gross Motor Function, Spasticity, Healthtron, Device, Cerebral Palsy.

\section{INTRODUCTION}

Cerebral palsy (CP) describes a group of permanent disorders, affecting the development of movement and posture, causing activity limitation, that are attributed to non-progressive disturbances that occurred in the developing fatal or infant brain (Rosenbaum et al., 2007). The motor disorders of $\mathrm{CP}$ are often accompanied by disturbances in sensation, perception, cognition, communication, behaviour and secondary musculoskeletal problems (Rosenbaum et al., 2007). CP has been classified into three different subtypes based on presenting motor impairments, i.e. spastic, dyskinetic or the ataxic form (Surveillance of Cerebral Palsy in Europe, 2000). Spasticity 
is the most prevalent form of dystonia (Hagberg, 2001). A broad diversity of muscle function impairments can be present in spastic paresis. Clinical symptoms of impaired muscle function can either be related to an impairment of muscle activation, leading to both deficit symptoms (e.g. paresis, loss of voluntary selective motor control) and excess symptoms (e.g. spasticity), or to a change in biomechanical properties of muscles and connective tissues (Becher et al., 1998)

The high voltage Healthtron device (HD) also referred to as electrostatic or electric field therapy apparatus is an attenuated alternating current device which serves to treat the human body in an electric field (Muri and Miyazaki,

1990). The HD has a conventional input voltage of 220-

$240 \mathrm{~V}$ and a high output voltage of $0-7000 \mathrm{~V}$ and operates at a frequency of 50-60Hz (Muri and Miyazaki, 1990). The machine has a chair with two separate foot electrodes which are connected to the attenuated high voltage machine. The feature of this electric field therapy apparatus is said to be easily effected all over the human body without current being applied directly to the human body (United States Patent, 2009).

In recent developments, electro-motor stimulation by means of functional electrical stimulation for improving motor function in children with spastic $\mathrm{CP}$ has been more widely used in clinical settings and has received more attention in the research literature (Carmick, 1993; Carmick, 1995; Comeaux et al., 1997, Kerret et al., 2004) although, the evidence available to support these current practice is limited and equivocal. (Barry, 2006; Patrick et al., 2001) On the other hand, the use of high voltage electric current devices in the management of chronic diseases remains controversial, though appears to be gaining wider acceptance in Asia. Several claims have been made by manufacturers on the therapeutic benefits of these devices on $\mathrm{CP}$ and several other disease conditions (Muri and Miyazaki, 1990). However, only a few studies have been carried out to test these claims (Miura et al., 2001; Onigbinde and Adedoyin, 2010).

There are generally very limited clinical trials on the efficacy of the HD. Furthermore, the claims of therapeutic efficacy of HD in alleviating spasticity and improving motor function to enhance developmental milestones in $\mathrm{CP}$ children is yet to be tested. This study was therefore designed to investigate the therapeutic efficacy of the HD on children with CP. It was hypothesized that HD treatment combined with conventional physiotherapy would improve motor function and reduce spasticity in children with CP after 4 weeks of treatment.

\section{METHODS}

\section{Study Participants}

Participants were children with CP attending the out-patient clinic of the Department of Physiotherapy, Lagos University Teaching Hospital, Idi-Araba, Lagos. Participants who fit the inclusion/exclusion criteria were approached for enrolment.

\section{Inclusion criteria were:}

(1) Diagnosis of spastic CP (quadriplegic type) - defined as generalised muscle hypertonia, poor to fair neck control and a gross motor function classification within level II and V as described by the gross motor function classification system (GMFCS) (Russel et al., 2002).

(2) Age 1-12 years.

\section{Exclusion criteria were:}

(1) Non-cooperative and non-tolerant participants to testing procedures during screening.

(2) Participants with fixed musculoskeletal deformities such as ankylosed joints.

(3) Previous treatment with HD.

(4) Participants with MAS rating of 4 i.e. limbs rigidly restricted in flexion or extension

The study was designed as a randomised controlled study. A total of 24 participants were involved in the study. Participants were randomly assigned into two groups HD and control (CON) groups. Each group had 12 participants. Ethical approval for the study was sought from the local ethics committee of the Lagos University Teaching Hospital. Informed consents were also sought from participants' parents/guardians.

\section{Outcome Measures}

\section{Gross Motor Function Measure-66 Motor Function}

Gross motor function of all participating children was assessed using the Gross Motor Function Measure-66 (GMFM-66); a modification of the GMFM-88 (Russel at al., 2000; Russel et al., 2002). The GMFM-88 is a standard criterion-referenced measure for detecting and monitoring changes in motor functions (Vos-Vromans et al., 2005). The 88 items of the test assess activities in five dimensions. Each item is scored using a 4-point Likert scale (0-3 with 0 representing the lowest performance level and 3 the highest). The GMFM-66 uses 66 of the 88 items and was developed using Rasch analysis to improve the sensitivity and interpretability of the test (Russel et al., 2002). For each child the values of the GMFM-88 was analyzed using the Gross Motor Ability Estimator computing scoring program to acquire an interval-level GMFM-66 score ranging from 0 to 100 (maximum score) which represents a child's overall level of gross motor functions in a quantitative form (Russel et al., 2002; Vos-Vromans et al., 2005). The GMFM-66 is a valid and reliable measure with responsiveness to change (Russel et al., 2002).

To evaluate the development of gross motor function in 4 weeks, the interval-level GMFM-66 scores of the first measurement pre-intervention was deducted from the GMFM-66 scores of the second measurement postintervention. The result of this deduction was recorded as the GMFM-66 change score and reflects the change in GMFM-66 score in 4 weeks (i.e. the progression or 
regression of gross motor functioning in 4 weeks after intervention).

\section{Modified Asworth Scale (MAS) Spasticity}

Spasticity of a muscle group that is most relevant for gross motor function in the upper extremity was assessed with the MAS adopting the evaluation rating described by Mutlu et al. (2008). The MAS measure spasticity and is applied manually to determine the resistance of muscle to passive stretching. The MAS needs no equipment and is commonly used in clinics (Bohannam and Smith, 1987; Young, 1994; Fosang et al., 2003). The intra-rater and interrater reliability of the MAS have been documented (Bohannam and Smith, 1987; Fosang et al., 2003; Cloptan et al., 2005).

The biceps muscles were assessed to test for spasticity; test movement (passive) was done in the direction of elbow extension from flexion. Test movement was performed over a duration of about 1 second (by counting "one thousand one"), as described by Bohannon and Smith (1987) in order to ensure consistency and reliability in measurements.

A separate recording sheet was used for each subject for both outcome measures so that the test results would not influence subsequent test results.

\section{Procedure}

Demographic data of all participants were gotten preintervention. Gross motor function and spasticity of all participant were also assessed pre-intervention and after 4 weeks (12 sessions) of treatment intervention.

A pilot study was performed to in order for the physiotherapists and assistants to be familiar with the HD and outcome measures.

Participants were randomly assigned into 2 groups HD group and CON group. The HD group had conventional physiotherapy including passive mobilisation exercises to all limbs, neck and trunk mobilisation/tactile stimulation and weight bearing exercises, followed by the HD treatment. The HD treatment involved the participant sitting on the device either alone or with support (on the parent/guardian's laps) for time period of 30 minutes (per session) and voltage was regulated as advised by the manufacture. The device was set at 3,000V and 5,000V for the first and second weeks respectively and at $7,000 \mathrm{~V}$ for subsequent weeks. The CON group received conventional physiotherapy as aforementioned. Participants in both groups had a total of 12 treatment sessions of 3 times a week for 4 weeks. Outcomes were assessed pre and post-intervention to determine any therapeutic effects of the HD on participants.

\section{STATISTICALANALYSIS}

SPSS 15 for windows package programme was used to analyse data. All demographic and quantitative data were expressed as mean \pm standard deviation $\left(\mathrm{X}^{-} \pm \mathrm{SD}\right)$ using descriptive statistics where appropriate. Paired sample t-test was used to detect changes in quantitative outcome data pre- and post-intervention within the HD and CON group. Wilcoxon signed rank test was used to test for significance in the MAS measurements within the groups. Independent sample t-test was used to compare quantitative values between the groups while Mann Whitney-U test was used to compare the qualitative values (from the MAS) between the two groups.

\section{RESULTS}

The study included 15 male and 9 female children; a total of 24 children with spastic quadriplegic CP (Table 1). The functional level of participants was classified according to the gross motor function classification system (GMFCS) (Russell et al., 2002). The number of participants in each category of $\mathrm{CP}$ classification is as presented in table 1 .

Table 1: Demographic Characteristics of Participant.

\begin{tabular}{lll}
\hline $\begin{array}{l}\text { Demographic } \\
\text { Characteristics }\end{array}$ & $\begin{array}{l}\text { HD Group } \\
(\mathbf{n}=\mathbf{1 2})\end{array}$ & $\begin{array}{l}\text { CON Group } \\
(\mathbf{n}=\mathbf{1 2})\end{array}$ \\
\hline Age (year) & $4.67 \pm 3.78$ & $4.53 \pm 3.64$ \\
Gender (male/female) & $7 / 5$ & $8 / 4$ \\
GMFCS & & \\
Level III & 2 & - \\
Level IV & 4 & 4 \\
Level V & 6 & 8 \\
\hline
\end{tabular}

HD Healthtron

Device

CON - Control

GMFCS Gross Motor Function Classification System

Changes in the pre- and post-test outcome measurements within the two groups are as presented in table 2. Significant improvement was noted in the GMFM-66 and the MAS in the HD and CON groups, except in the MAS measurement for the CON group.

Table 2: Changes in Outcome Measures within each Group Pre- and Post-treatment.

\begin{tabular}{lll}
\hline & $\begin{array}{l}\text { GMFM-66 }(\%) \\
\mathbf{X}^{-} \pm \mathbf{S D}\end{array}$ & $\begin{array}{l}\text { MAS } \\
\mathbf{X}^{-} \pm \mathbf{S D}\end{array}$ \\
\hline HD Group & & \\
Pre-Rx & $11.98 \pm 2.36$ & $3.00 \pm 0.60$ \\
Post-Rx & $20.34 \pm 4.69$ & $1.17 \pm 1.12$ \\
t-value/z-value & -6.07 & -3.12 \\
Mean rank & - & 6.50 \\
p-value & $<0.001 *$ & $0.002 *$ \\
CON Group & & \\
Pre-Rx & $13.79 \pm 3.59$ & $3.33 \pm 0.65$ \\
Post-Rx & $16.64 \pm 4.04$ & $3.08 \pm 0.67$ \\
t-value/z-value & -4.02 & -1.73 \\
Mean rank & - & 2.00 \\
p-value & $0.02 *$ & 0.083 \\
\hline
\end{tabular}

*Differences between the two groups; significant at $\mathrm{p}<0.05$ GMFM Gross Motor Function Measure

MAS Modified Asworth Scale

Rx Treatment

HD Healthtron

Device

CON Control 
Comparison of outcome measures (as measured by the GMFM-66 and MAS) between the HD and the CON groups after 4 weeks of treatment intervention revealed a superior improvement in spasticity and gross motor function in the HD group over the CON group.

Table 3: Changes in Outcome Measures after Intervention

\begin{tabular}{llll}
\hline & $\begin{array}{l}\text { HD Group } \\
\mathbf{X}^{-} \pm \text {SD }\end{array}$ & $\begin{array}{l}\text { CON Group } \\
\mathbf{X}^{-} \pm \text {SD }\end{array}$ & P-value \\
\hline GMFM-66(\%) & $8.35 \pm 4.77$ & $2.85 \pm 2.46$ & $0.02 *$ \\
MAS & $1.83 \pm 0.72$ & $0.25 \pm 0.45$ & $<0.001 *$ \\
\hline
\end{tabular}

*Differences between the two groups; significant at $\mathrm{p}<0.05$ GMFM Gross Motor Function Measure

MAS Modified Asworth Scale

HD Healthtron Device

CON Control

\section{DISCUSSION}

In the present study, changes in gross motor function and spasticity were examined in a sample of children with CP. The internationally well acknowledged GMFM-66 was used as the outcome measure for gross motor function and MAS for spasticity. A significant improvement in gross motor function was found in both groups and significant reduction in spasticity was only seen in the HD group. Comparison of changes in outcome measures between the HD and CON groups revealed that the HD group was significantly superior to the CON group as evaluated by the two outcome measures, especially in the reduction of spasticity.

Although the efficacy of the HD is still inconclusive, there is a growing body of evidence supporting the claims made by its manufacturer (Muiru et al., 2001; Onigbinde and Adedoyin, 2010). Ongbinde and Adedoyin (2010) in a recent study documented the potency of the HD in lowering blood pressure and fasting blood sugar. To the best of our knowledge, this is the very first randomised controlled study to investigate the therapeutic efficacy of the $\mathrm{HD}$ on children with $\mathrm{CP}$. At the moment there are generally few studies on the HD and its awareness and clinical application in Nigeria and Africa is still relatively low compared to Asia.

The reduction in spasticity in $\mathrm{CP}$ children in the HD group during the study was quite remarkable towards the end of the study (last 3 sessions). This may be attributed to a probable increase in body metabolism induced by the HD during treatment as described by Muiru et al. (2001). However, the physiological mechanism by which the HD evokes changes in the human body to bring about therapeutic effects is not fully known.

Improvement in gross motor function has been indicated after periods of intensive physiotherapy for nonambulatory children (Alh et al., 2002), and in children who have practiced functional tasks intensively in their everyday environments (Trahan and Malouin, 2005). Results from this study support this submission. Change in the GMFM-66 score was found to be remarkably significant not just in the HD but also in the CON group (p $<0.05$ ) indicating improvement in gross motor function in the CON group in which participants had only conventional physiotherapy. Although a few participants in the CON group had improvement in the MAS score; it was not significant enough in overall analysis $(\mathrm{p}=0.083)$. An average improvement of about $10 \%$ and $3 \%$ were recorded for gross motor function in the $\mathrm{HD}$ and $\mathrm{CON}$ groups respectively. This result despite been significant in both groups did not translate into much functional independence in the participants. The GMFM-66 evaluates and reports gross motor function in percentage scores of 0 to 100 . Most of the participants irrespective of group had an increase in the GMFM-66 score and the highest score was $36.79 \%$ in the HD group which is still not up to $50 \%$ of gross motor function. A longer period of treatment in terms of sessions may be needed to achieve a better outcome.

A time duration of 30 minutes per session was employed in this study with voltage intensity ranging from $3,000-7,000 \mathrm{~V}$ and this was effective enough to modulate spasticity and improve gross motor function in children with CP. Treatment time and intensity of the HD were not documented in a previous study (Onigbinde and Adedoyin, 2010) hence comparison could not be made. We suggest that future studies clearly document this in order to conclusively determine the dose-response effect of this device.

$\mathrm{CP}$ is the most common cause of physical disability affecting children. The spastic form of $\mathrm{CP}$ is most common in these patients and additional clinical sign may include muscle shortening, diminished and uncoordinated voluntary control. In most centres, management of spasticity in CP patients is left to the physiotherapist while in few other centres, medications and surgery are employed. Muscle weakness, depression of the central nervous system and respiration have been reported to be associated with the use of drugs (e.g botulinum toxin) in the treatment of spasticity (Katz and Campagnolo, 1994). Furthermore, severe post-operative pain, spinal deformity, increased incidence of spondylolisthesis and potential for recurrence of spasticity have been documented to be associated with rhizotomy (McDonald and Hays, 1994). Conversely, the HD has been reported to be relatively risk free (Muri and Miyazaki, 1990; Muira et al., 2001; Onigbinde and Adedoyin, 2010)

The primary modality in the arsenal of physiotherapists is exercise therapy in the form of passive mobilisation. Others modalities used are cryotherapy and neuromuscular electrical stimulation. Although studies have shown the efficacy of these modalities on spasticity in children with CP (Scheker et al., 1999; Akinbo et al., 2007) they are seldom used. The most conveniently and frequently used is passive mobilisation. However, passive mobilisation appears not to be enough to combat the clinical challenge posed by spasticity in CP patients. The results 
from the present study suggests that conventional physiotherapy combined with HD therapy may serve as a good treatment option in modulating spasticity and progressively improving function in children with $\mathrm{CP}$.

There may be a need for the manufactures to specially design a paediatric version of the HD so as to allow paediatric patients sit independently on the HD chair. The mothers/guardians of participants involved in the study through carrying of participants who were not grown and balanced enough to sit by themselves reported fatigue and sleepiness after each treatment session, especially during the last few sessions. This corroborates reports from Onigbinde et al. (2010) where fatigue and hunger where reported by participants after each treatment session with HD. This may also be a contributing factor to the reduction in spasticity observed in the study.

It is imperative to emphasize the limitations of this present research and in essence make recommendations for further studies. A major issue is the paucity of information on the physiological effects of the HD on the human body. Although some studies have reported HD to have little to no side effects (Muira et al., 2001; Onigbinde and Adedoyin, 2010), the mechanism by which it induces its therapeutic effects is not fully known. It is therefore necessary that studies are carried out at both clinical and laboratory settings to further investigate the therapeutic effects of HD on specific non-communicable diseases and the physiological activities it evokes in the human body. It is also important to investigate the sustainability of the therapeutic benefits of the HD.

\section{CONCLUSION}

Attenuated high voltage current generated from the HD combined with conventional physiotherapy resulted in a significant improvement in gross motor function and reduction in spasticity in children with $\mathrm{CP}$. A combination of the two modalities was found to be significantly superior to conventional physiotherapy alone. The HD may serve as an adjunct electrotherapy device with other physiotherapy modalities in improving function and modulating spasticity in children with $\mathrm{CP}$. We recommend that the long term effects of the HD on children with $\mathrm{CP}$ be investigated in future studies. Furthermore, there is an urgent need for laboratory studies to fully understand the physiological and biochemical effects of the HD on the human body.

\section{ACKNOWLEDGEMENT}

This study was supported by a grant from the LATO Nigerian Limited, Ibadan. The research team wish to sincerely thank the entire company and specifically the chairman/CEO of LATO Nigerian Limited; we appreciate your overall support. We also wish to profoundly thank the parents of all the children who voluntarily participated in the study.

\section{REFERENCES}

- Ahl LE, Johansson E, Granat T, Carlberg EB (2005). Functional therapy for children with cerebral palsy: an ecological approach. Dev Med Child Neurol. 47: 613-619.

- Akinbo SRA, Tella BA, Otunla AB, Temiye EO (2007). Comparison of the effect of Neuromuscular Electrical Stimulation and Crotherapy on Spasticity and Hand Function in patients with Cerebral Palsy. Nigerian Medical Practitioner. 51(6): 128-132

- Barry MJ(1996). Physical therapy interventions for patients with movement disorders. J Child Neurol. 11: S51-S60.

- Becher JG, Harlaar J, Lankhorst GJ, Vogelaar TW (1998): Measurement of impaired muscle function of the gastrocnemius, soleus, and tibialis anterior muscles in spastic hemiplegia: A preliminary study. J Rehabil Res Dev. 35: 314-26.

- Bohannan RW, Smith MB (1987). Interrater reliability of a modified ashworth scale of muscle spasticity. Phys Ther. 67: 206-207.

- Boyd RN, Graham HK (1999). Objective measurement of clinical findings in the use of botulinum toxin type a for the management of children with cerebral palsy. Eur J Neurol 6: S23-S35.

- Carmick J (1993). Clinical use of neuromuscular electrical stimulation for children with cerebral palsy, 1: lowerextremity. Phys Ther. 73: 505-513.

- Carmick J (1995). Managing equinus in children with cerebral palsy: electrical stimulation to strengthen the triceps surae. Dev Med Child Neurol. 37: 965-975.

- Clopton N, Dutton J, Featherston T, Grigsby A, Mobley J, Melvin J (2005). Interrater and intrarater reliability of the modified ashworth scale in children with hypertonia. Pediatr Phys Ther. 17:268-274.

- Comeaux P, Patterson N, Rubin M, Meiner R (1997). Effect of neuromuscular electrical stimulation during gait in children with cerebral palsy. Pediatr Phys Ther. 9: 103-109.

- Fosang AL, Galea MP, McCoy AT, Reddihough DS (2003). Measures of muscle and joint performance in the lower limb of children with cerebral palsy. Dev Med Child Neurol. 45: 664-70.

- Hagberg B, Hagberg G, Beckung E, Uvebrant P (2001). Changing panorama of cerebral palsy in sweden. VIII. prevalence and origin in the birth year period 1991-94. Acta Paediatr. 90: 271-7. 
- Katz RT, Campagnolo DI (1994). Pharmacologic Management of Spasticity. Physical Medicine and Rehabilitation. State of the Art Reviews. 8: 473-480.

- Kerret C, McDowell B, McDonough S (2004). Electrical stimulation in cerebral palsy: a review of effects on strength and motor function. Dev Med Child Neurol. 46: 205-213.

- McDonald CM, Hays RM (1994). Selective Dorsal Rhizotomy: Patient selection, Intraoperative Electrophysiologic Monitoring and Clinical Outcome. Physical Medicine and Rehabilitation: State of the Art Reviews 8:579-604.

- Miura N, Shusaku, S, Yuji H (2001). Effects of Exposure to Alternating Curreent, High Voltage, Electric Potential on Experimental Hypertension in Rats. Journal of the Japan Vetinary Medical Association. 54:6, 472-475.

- Muri and Miyazaki (1999). Clinical Report Manual on Healthtron Machine.

- Mutlu A, Livanelioglu A, Gunel MK (2008). Reliability of Ashworth and Modified Ashworth Scales in Children with Spastic Cerebral Palsy. BMC Musculoskeletal Disorders 9: 44.

- Onigbinde AT, Adedoyin RA (2010). Effect of High Voltage Healthtron Device on Blood Pressure and Plasma Glucose of Normal Subjects. Indian Journal of Physiotherapy and Occupational Therapy. 4(1): 29-31.

- Patrick JH, Roberts AP, Cole GF (2001). Therapeutic choices in the locomotor management of the child with cerebral palsy: more luck than judgement? Arch Dis Child. 85: $275-279$.

- Rosenbaum P, Paneth N, Leviton A, Goldstein M, Bax M, Damiano D, et al. (2007). A report: The definition and classification of cerebral palsy April 2006. Dev Med Child
Neurol Suppl. 109: 8-14.

- Russell DJ, Avery LM, Rosenbaum PL, Raina PS, Walter SD, Palisano RJ (2000). Improved scaling of the gross motor function measure for children with cerebral palsy: Evidence of reliability and validity. Phys Ther. 80: 873-85.

- Russel DJ, Rosenbaum PL, Avery LM, Lane M (2002). Gross Motor Function Measure (GMFM-66 \& GMFM88) User's Manual. Mac Keith Press, London.

- Surveillance of Cerebral Palsy in Europe (SCPE) (2000). Surveillance of Cerebral Palsy in Europe: a collaboration of cerebral palsy surveys and registers. Dev Med Child Neurol. 42: $816-824$.

- Scheker LR, Chester SP, Ramirez S (1999). Neuromuscular Electrical Stimulation and Dynamic bracing as a treatment for upper extrimity spasticity in Children with Cerebral Palsy. Br J Hand Surg. 2: 222-232.

- Trahan J, Malouin F (2002). Intermittent intensive physiotherapy in children with cerebral palsy: a pilot study. Dev Med Child Neurol. 44: 233-239.

- United States Patent (2009). http:/ www.freepatentsonline.com/4802470.html

- Vos-Vromans DC, Ketelaar M, Gorter JW (2005). Responsiveness of evaluative measures for children with cerebral palsy: The gross motor function measure and the pediatric evaluation of disability inventory. Disabil Rehabil 27: $1245-52$.

- Wright FV, Rosenbaum PL, Goldsmith CH, Law M, Fehlings DL (2008). How do changes in body functions and structures, activity, and participation relate in children with cerebral palsy? Dev Med Child Neurol. 50: 283-9.

- Young RR (1994). Spasticity: A review. Neurology. 44(119): $12-20$. 